\title{
LOS MOVIMIENTOS SOCIALES LATINOAMERICANOS TEORÍAS CRÍTICAS Y DEBATES SOBRE LA FORMACIÓN
}

Mariana Fry

\section{Resumen}

El artículo revisa los abordajes críticos sobre movimientos sociales desarrollados en las últimas dos décadas en América Latina, buscando discutir hasta qué punto estos suponen una renovación teórica en el campo de estudios sobre movimientos sociales. Para ello, se repasan las críticas formuladas a las teorías europeas y norteamericanas en esta materia, las diversas formas de integración y reelaboración de elementos provenientes de estos enfoques, y los debates y conceptos formulados específicamente para entender las características de la movilización social desplegada en nuestro continente. En este marco, se presta especial atención al lugar que ocupa en la producción académica actual el debate en torno a la formación realizada desde los movimientos sociales.

Palabras clave: movimientos sociales, teorías latinoamericanas, formación.

\section{Abstract}

Latin American social movements. Critical theories and debates about training

This article reviews the critical approaches on social movements developed in the last two decades in Latin America, aiming to discuss to which extent these involve a theoretical renewal in the field of study of social movements. For this sake, we review the criticism to European and North American theories, the different ways of integration and reworking of elements from these approaches, and the debates and concepts specifically elaborated to understand the features of Latin American social mobilization. In this context, the place of the debate around training imparted by social movements in the current academic production is given special attention.

Keywords: social movements, Latin American theories, training.

\footnotetext{
Mariana Fry: Candidata a doctora por el Programa de Doctorado en Sociología de la Facultad de Ciencias Sociales, Universidad de la República. Se desempeña como docente del Departamento de Sociología de la Facultad de Ciencias Sociales de la Universidad de la República (Uruguay).

ORCID iD: 0000-0003-0937-3033

Email: mariana.fry@cienciassociales.edu.uy
} 


\section{Introducción}

En las últimas dos décadas los estudios sobre movimientos sociales y acciones colectivas han tenido un amplio desarrollo en América Latina, que supuso la proliferación de trabajos empíricos y debates teóricos. Esta producción académica tuvo como trasfondo el despliegue de un nuevo ciclo de luchas en la región, cuyos primeros hitos pueden ubicarse en el levantamiento zapatista de 1994 y en la guerra del agua desatada en Bolivia a comienzos del siglo XXI.

A diferencia de la discusión sobre el tema registrada en la década de los ochenta, cuando se introdujo en la región el concepto de movimiento social, en la actualidad varios trabajos cuestionan la incorporación acrítica de teorías formuladas para pensar otros contextos y señalan la necesidad de elaborar categorías analíticas propias, que contemplen la especificidad de la región.

El presente artículo se propone revisar y valorar las principales contribuciones realizadas desde América Latina para el estudio de los movimientos sociales y acciones colectivas en las últimas dos décadas, es decir, el conjunto de trabajos que pretenden dar cuenta del "cambio de época" de los movimientos sociales latinoamericanos ${ }^{1}$ (Svampa, 2008). Desde el punto de vista teórico, se retoma el debate acerca de si es posible hablar de un paradigma latinoamericano sobre movimientos sociales, el cual fuera planteado por Gohn (1997) en los años noventa, buscando actualizarlo a la luz de los desarrollos recientes.

Para dar cuenta de esta discusión, el artículo analiza específicamente los abordajes críticos contemporáneos, es decir, aquellos que cuestionan la aplicabilidad de las teorías producidas en otros contextos y piensan los movimientos sociales asumiendo a América Latina como perspectiva de análisis (Falero, 2020). En este marco, se presta especial atención al lugar que han ocupado en la producción académica actual los debates en torno a la educación y la formación realizadas desde los movimientos sociales.

El artículo comienza revisando los abordajes clásicos en el estudio de los movimientos sociales y acciones colectivas, prestando especial

1 Dada la ausencia de trabajos que sistematicen los desarrollos en la materia en los distintos países y la imposibilidad de realizar una labor más exhaustiva que permita abarcar a la totalidad de la producción en el área, se optó aquí por revisar los trabajos que sistematizan esta discusión, complementando esta búsqueda con una revisión propia que recoge los aportes de autores y estudios relevantes. Naturalmente, la búsqueda podría ser profundizada, pero en su estado actual comprende un panorama general del debate contemporáneo sobre el tema. 
atención al modo en que estos fueron incorporados en nuestro continente. Posteriormente, repasa la producción teórica latinoamericana acerca del tema a partir de la década de los ochenta del siglo pasado, cuando se introdujo la noción de movimiento social. A partir de aquí se hace foco en la producción teórica y empírica desarrollada en las dos últimas décadas, mostrando los debates y conceptos que esta abrió y los diálogos que estableció con los abordajes clásicos sobre el tema. Finalmente, se presenta la discusión sobre la formación en los movimientos sociales y su relación con los debates del llamado "cambio de época". Para cerrar, se colocan algunas reflexiones acerca de los límites y alcances de la renovación teórica analizada.

\section{Las teorías clásicas sobre movimientos sociales}

Hacia fines de los años sesenta diversos acontecimientos sacudieron al mundo. Desde las protestas de 1968 en Francia hasta los movimientos antibelicista y feminista en Estados Unidos, un conjunto de formas novedosas de movilización comenzaba a mostrar que la política ya no podía leerse exclusivamente desde las formas clásicas de sindicato y partido. En este contexto comienzan a desplegarse, tanto en Europa como en Estados Unidos, los estudios sobre movimientos sociales. ${ }^{2}$ Estos pueden agruparse en tres grandes cuerpos teóricos. ${ }^{3}$

En primer lugar, puede identificarse la teoría de la movilización de recursos desarrollada en Estados Unidos, a partir de fines de los años sesenta, por autores como Olson, McCarthy y Zald (Gohn, 1997). Desde un enfoque utilitarista de la acción, esta escuela se propone entender a los movimientos sociales como grupos de interés, centrando el análisis en su capacidad de movilización de recursos — humanos, financieros, de infraestructura-. Si bien esta teoría tuvo un gran impacto en Estados Unidos, su llegada a Europa y América Latina fue escasa. De acuerdo con Alonso (2009), esta mirada produjo rechazo entre los pensadores de izquierda, dado que aplicaba un análisis organizacional al estudio de los movimientos sociales, llegando al punto de compararlos con empresas.

La teoría del proceso político surge en la década de 1970 en Estados Unidos, a partir de los trabajos de Tilly, Tarrow y McAdam. Desde esta

2 Si bien existían trabajos previos, estos tenían un escaso nivel de desarrollo teórico y empírico (Della Porta y Diani, 2015), a la vez que se centraban en el estudio de grupos de presión y acciones colectivas puntuales, más que en movimientos sociales propiamente dichos (Gohn, 1997).

3 La delimitación de tres grandes teorías es la más habitual en la literatura especializada (véase, por ejemplo, Alonso, 2009; Diani, 2015; McAdam, McCarthy y Zald, 1999; entre otros). Sin embargo, esta mirada puede complejizarse. Autoras como Gohn (1997) subdividen la escuela europea en dos vertientes, una de impronta más culturalista ligada a los aportes de Melucci y Touraine, y otra neomarxista en la que se incluyen los trabajos de Castells y de historiadores como Hobsbawm y Thompson. 
perspectiva se critica fuertemente el enfoque anterior, destacándose la importancia de estudiar el contexto político en que la acción colectiva se desarrolla y analizar los factores que fomentan o desincentivan la emergencia de movimientos sociales, los cuales componen la "estructura de oportunidades políticas" (Gohn, 1997). En sus primeros años, este enfoque compartió el protagonismo con la teoría de la movilización de recursos, pero con el paso del tiempo terminó eclipsándola (Alonso, 2009). En Europa logró una influencia importante, mientras que su llegada a América Latina fue tardía, comenzando a adquirir relevancia recién en la década de $1990 .{ }^{4}$ En la actualidad, la teoría del proceso político ha adquirido relevancia en los estudios latinoamericanos. La compilación realizada por Almeida y Cordero (2017) resulta un buen ejemplo de ello.

La escuela de los nuevos movimientos sociales (en adelante, NMS) surge en Europa a fines de los años sesenta y reúne los aportes de autores como Touraine y Melucci. Desde esta perspectiva se destaca la importancia de los aspectos culturales que dan sentido a la acción colectiva (Touraine, 1990), a la vez que se prioriza el estudio de los procesos de construcción de identidades desplegados por los movimientos sociales (Melucci, 1994). Los autores que se incluyen en este enfoque han coincidido en señalar la emergencia de NMS que se diferencian del movimiento sindical, caracterizados por no constituirse a partir de una clara base clasista, centrar sus demandas en aspectos más culturales y menos económicos, y organizarse con formatos más laxos y descentralizados (Gohn, 1997). Esta teoría fue la más influyente en América Latina, particularmente durante las décadas de 1980 y 1990. La influencia de Touraine fue muy importante en los años ochenta, mientras que en los noventa los trabajos de Melucci fueron adquiriendo mayor relevancia (Alonso, 2009).

Hasta el inicio de la década de 1980, el debate entre escuelas dominó las discusiones en el campo de los estudios sobre movimientos sociales y acciones colectivas. Algunos autores decodificaron esta polémica como la disputa entre el paradigma identitario y el de la acción estratégica, sintetizando en esta última corriente la contribución de las dos escuelas norteamericanas (Cohen, 1985). La teoría de los NMS fue criticada por desconocer la importancia de las relaciones entre movimientos sociales y política institucional, mientras que las teorías norteamericanas fueron acusadas de dar por sentados los

4 Analizando el desarrollo de los estudios sobre movimientos sociales en Brasil, Bringel (2011) señala que en ese país la recepción de las teorías norteamericanas fue indirecta, tardía y limitada. Indirecta porque muchos autores relevantes fueron leídos a partir de trabajos realizados por investigadores brasileños, tardía porque la traducción de los textos claves de esta teoría fue realizada más de una década después de su aparición, y limitada porque muchos trabajos relevantes aún no han sido traducidos ni debatidos a nivel local. Se sostiene aquí que esta lectura podría extenderse para analizar los estudios sobre movimientos sociales —al menos - en el cono sur de América Latina, donde la influencia de este enfoque comienza a registrarse a fines de los años noventa. 
procesos de construcción y atribución de sentido que permiten producir la acción colectiva (Melucci, 1994).

En los años posteriores, el diálogo entre estos enfoques comenzó a darse en forma cada vez más fluida, de manera que en la actualidad resulta artificial hablar de distintos paradigmas, más bien deberíamos hablar de perspectivas teóricas con énfasis diferenciados, pero también con importantes niveles de intercambio (McAdam, McCarthy y Zald, 1999).

Como consecuencia de ello, hacia fines de la década de 1980, el debate en torno a los procesos de construcción de identidad desarrollados por los movimientos sociales empezó a adquirir centralidad entre los teóricos norteamericanos. A partir de los trabajos pioneros de Snow y Benford, comenzaron a realizarse estudios orientados a analizar la construcción de marcos interpretativos compartidos que estructuran la percepción de la realidad y organizan la acción colectiva. Desde entonces, el frame analysis ha adquirido relevancia mundial (Benford y Snow, 2000). ${ }^{5}$ Sin embargo, su alcance en América Latina es limitado, posiblemente por el hecho de que la mayoría de las obras relevantes no han sido traducidas del inglés.

En este período se destaca también la emergencia de teorías de síntesis, que combinan en forma variada los distintos elementos presentes en los estudios sobre movimientos sociales, siendo una de las obras más destacadas la de Tarrow (2009). Asimismo, es indicativa de este proceso la publicación en 1992 de un trabajo de Diani que avanza hacia una definición de síntesis del concepto de movimiento social (Diani, 2015).

El advenimiento del siglo XXI trajo aparejados cambios en las formas de contestación social, entre los que se destacan la creciente burocratización de los movimientos sociales que habían dado origen a estas teorías y la emergencia de nuevas formas de activismo transnacional (Della Porta y Diani, 2015). En este escenario, se produjeron debates y reelaboraciones dentro de los distintos cuerpos teóricos.

Melucci (1999) profundizó en la idea de "sociedades complejas" e impulsó un viraje del estudio de los movimientos sociales al estudio de la sociedad civil, entendida como espacio de disputa por la democratización de la vida cotidiana en contextos de globalización. Esto tuvo importantes impactos en los estudios sobre los movimientos sociales desarrollados en América Latina, donde se observó una proliferación de investigaciones que apuntaban a conceptos como participación y democracia (Alonso, 2009).

5 Si bien este enfoque constituye una perspectiva articulada y coherente, que arroja luz sobre aspectos novedosos en el estudio de los movimientos sociales, no lo ubicamos aquí como un paradigma, ya que este puede conceptualizarse como un desarrollo dentro de las corrientes norteamericanas (Benford y Snow, 2000). 
Por su parte, el enfoque del proceso político se encaminó hacia una profunda revisión que resultó en una redefinición de su objeto de estudio. ${ }^{6}$ En vez de la tríada "movimientos sociales, revoluciones y acción colectiva", McAdam, Tilly y Tarrow propusieron centrar sus análisis en la "contienda política", buscando construir un dominio académico más amplio que permitiera entender las dinámicas de movilización social relacionando los movimientos sociales, los ciclos de protesta y las revoluciones entre sí, con la política institucional y con el cambio social (McAdam y Tarrow, 2011). La llegada de este enfoque a América Latina se dio de la mano de este proceso de revisión, lo que permitió reorientar la mirada hacia fenómenos como acciones colectivas y eventos de protesta. En consecuencia, los estudios específicos sobre movimientos sociales entraron en declive hacia finales del siglo $\mathrm{XX}$.

\section{Los estudios sobre movimientos sociales en América Latina}

En América Latina, los estudios sobre los movimientos sociales han tenido un desarrollo particular. Durante las décadas en que en Europa y Estados Unidos empezaba a desplegarse este campo de estudios, varios países latinoamericanos atravesaban procesos dictatoriales que reprimieron toda forma de movilización social. Es recién en la década de los ochenta, con el inicio de las transiciones hacia regímenes democráticos, cuando, lentamente, empezaron a emerger nuevas formas de asociación y movilización.

En este contexto comienzan a desarrollarse los estudios sobre movimientos sociales en la región, ${ }^{7}$ que desde el punto de vista teórico estuvieron fuertemente influenciados por la teoría europea de los NMS (Gohn, 1997). En este escenario, el debate latinoamericano estuvo orientado a analizar la emergencia de nuevos sujetos, como los movimientos de mujeres y jóvenes o las organizaciones vinculadas a la lucha por derechos sociales básicos (tierra, vivienda, servicios públicos), los cuales afloraron vigorosamente hacia el final de los regímenes dictatoriales.

Los estudios dedicados a los NMS señalaban la heterogeneidad de estas luchas y su carácter novedoso respecto a los actores clásicos del campo popular - básicamente, los movimientos de trabajadores y campesinos-. Mientras que los primeros estaban orientados por transformaciones de tipo cultural y se enfatizaba su carácter de productores de nuevas identidades, los

$6 \quad$ El principal hito en este proceso de renovación fue el proyecto "contentious politics". Este se propuso desarrollar una síntesis teórica y empírica de los estudios sobre la contienda política, según fue publicado por primera vez en 1996 en el texto "To map contentious politics", en la revista Mobilization: An International Journal (McAdam, Tarrow y Tilly, 2009), y tuvo como resultado la publicación del libro Dynamics of Contention (McAdam, Tarrow y Tilly, 2001).

$7 \quad$ Si bien existen debates previos que abordan el problema del sujeto colectivo, la introducción del concepto de movimiento social responde a este período (Falero, 2018). 
segundos eran ubicados por la literatura especializada en torno a demandas de corte material, ligados a una dinámica de lucha de clases (Falero, 2018). ${ }^{8}$

Si bien la tónica general del período es la aplicación de la teoría europea de los NMS a partir de autores como Touraine, Melucci y Castells, se identifican algunos esfuerzos de adaptación interesantes que vinculan estos debates con las particularidades de la región. Ejemplo de ello son los trabajos de Camacho (1987), que acuñan el concepto de "movimientos populares" para referirse a las características específicas que estos fenómenos adquieren en nuestro continente.

Los años noventa estuvieron caracterizados por un declive de la importancia de los estudios realizados en esta temática, tal como se adelantó en el apartado anterior. En América Latina este proceso estuvo asociado al debilitamiento de los actores colectivos "clásicos" bajo la imposición del modelo neoliberal, junto con una pérdida de importancia de los sujetos que habían eclosionado luego de las dictaduras. En este escenario, adquirieron relevancia nociones como la de sociedad civil ${ }^{9}$ (Alonso, 2009; Falero, 2018), que retomaban las reelaboraciones procesadas en el interior de la teoría de los NMS, descentrando el análisis del concepto de "movimientos sociales".

De acuerdo con Svampa (2009), hacia fines de esta década comenzaron a surgir nuevas formas de acción colectiva, más fragmentadas y ligadas a demandas puntuales, que asumían la acción directa como vía principal. ${ }^{10}$ Estas transformaciones posibilitaron el surgimiento de estudios que incorporaban la noción de protesta como referencia central, retomando aportes provenientes de las teorías norteamericanas. ${ }^{11}$ Esto se dio en un escenario en el que la influencia de este enfoque se expandía en la región, tal como se comentó en el apartado anterior.

Uno de los trabajos más influyentes del período (Gohn, 1997) señalaba entonces que, si bien es posible identificar en la región un paradigma bien diferenciado de luchas, no existe un "paradigma latinoamericano" en el

8 Uno de los trabajos más relevantes de este período es la compilación realizada por Calderón (1986), que reúne estudios generales sobre los movimientos sociales en distintos países latinoamericanos, abarcando los casos de Argentina, Brasil, Uruguay, Bolivia, Ecuador, Perú, Colombia, Venezuela, Chile y Paraguay, además de incluir una síntesis elaborada por el compilador. Varios de los artículos que allí se recuperan ilustran los debates aquí planteados.

9 Cabe aclarar que, si bien el concepto de sociedad civil tiene en sus orígenes una matriz gramsciana, su utilización en América Latina en este período se vio despojada de su contenido crítico, en la medida en que se lo desvinculó de sus relaciones con las nociones de hegemonía y conflicto. De este modo, según Falero (2018) la idea de sociedad civil fue apropiada para referir a todo aquello que no es el Estado ni los partidos políticos, integrando desde organizaciones no gubernamentales hasta nucleamientos de empresarios o iglesias.

10 Piénsese, por ejemplo, en los cortes de ruta en Argentina o en los escraches realizados en Argentina y Uruguay.

11 Véase, por ejemplo, el trabajo de Schuster et al. (2006), que estudia la protesta social en Argentina en el período 1989-2003. 
estudio de los movimientos sociales, dado que los estudios realizados en nuestro continente suelen apoyarse en la utilización de teorías europeas y norteamericanas.

"Hablar de un paradigma teórico latinoamericano sobre movimientos sociales es más una colocación estratégica que real. Lo que existe es un paradigma bien diferenciado de luchas y movimientos sociales, en la realidad concreta, cuando se lo compara con los movimientos europeos, norteamericanos, canadienses, etc., y no un paradigma teórico propiamente dicho." ${ }^{\prime 2}$ (Gohn, 1997, p. 211).

Estas ideas ilustran con claridad el panorama en las décadas de 1980 y 1990. Sin embargo, sostenemos aquí que esta situación de debilidad teórica empieza a cambiar en el siguiente período.

A partir del año 2000 es posible constatar en América Latina una emergencia de luchas sociales diversas. Desde la guerra del agua y la guerra del gas en Bolivia, pasando por las movilizaciones zapatistas en México, hasta las luchas estudiantiles en Chile, nuestro continente se vio sacudido por distintas formas de protesta que ponían en cuestión los resultados del proyecto neoliberal implementado en la región. Esto despertó el interés de los analistas, que desde distintos ángulos centraron la mirada en las luchas sociales que germinaban en el continente.

En este escenario comenzaron a producirse nuevas teorizaciones, que integraron elementos provenientes de distintos enfoques para comprender las nuevas dinámicas de movilización y que, a nuestro entender, han contribuido a una renovación teórica de los estudios latinoamericanos sobre movimientos sociales. ¿Qué características tienen estas contribuciones? ¿Qué conceptos y debates han aportado? La sección que sigue pretende abrir este debate.

\section{Los abordajes críticos contemporáneos}

Resulta difícil reconstruir este campo de estudios por dos razones. En primer lugar, porque se trata de una producción reciente sobre la cual existen muy pocos trabajos de sistematización, y, en segundo lugar, porque es un campo extremadamente heterogéneo en cuanto a sus perspectivas teóricas.

En la investigación aquí realizada se identificaron algunas publicaciones que contribuyen a caracterizar esta producción. El artículo de Gohn (2008) reconoce cuatro abordajes contemporáneos en el estudio de los movimientos sociales, que se encuentran presentes en las investigaciones realizadas en América Latina. En primer lugar, las teorías centradas en ejes culturales basadas en los trabajos de Melucci y Touraine. En segundo lugar, las 
teorías enfocadas en la búsqueda de justicia social, apoyadas en los aportes de Honneth y Fraser. En tercer lugar, la autora identifica un conjunto de abordajes que parten de una perspectiva teórica crítica y destacan la capacidad de resistencia de los movimientos sociales y su lucha contra el neoliberalismo, donde adquieren centralidad las elaboraciones en torno a la autonomía. Finalmente, la autora ubica un cuarto eje que incluye estudios centrados en el proceso de institucionalización de las acciones colectivas, los cuales retoman los aportes de Tarrow, McAdam, McCarthy y Zald. La mirada específicamente latinoamericana es la que se recoge en el tercer eje y dialoga con las teorías que este artículo se propone revisar.

Por otra parte, se han publicado revisiones que parten de una perspectiva crítica, recogiendo específicamente aquellos desarrollos propios de América Latina y cuestionando la utilización de categorías provenientes de otros contextos. Así, Seoane, Taddei y Algranati (2011) critican la aplicación de abordajes de procedencia europea, que colocan el concepto de movimiento social en oposición al de movimiento obrero, para centrarse específicamente en el estudio de las miradas críticas latinoamericanas. Retomando elementos de la tradición marxista, se recuperan las visiones que ubican en el centro de la escena a los conflictos de clase y destacan el carácter popular de los movimientos sociales latinoamericanos.

En una línea similar, Modonesi e Iglesias (2016) recapitulan los desarrollos teóricos novedosos sobre los movimientos sociales latinoamericanos del cambio de siglo. Desde una perspectiva marxista crítica, los autores señalan que estos contribuyeron a desplazar la mirada utilitarista sobre los movimientos sociales y a reintroducir el conflicto como centro de la teoría social. Pese a identificar una importante producción teórica, sostienen que no se ha configurado un paradigma alternativo que pueda desplazar a los enfoques europeo y norteamericano, ya que no se ha desarrollado una reconstrucción teórica deliberada y sistemática.

Por último, Svampa (2009) señala que en América Latina se ha afianzado una tradición analítica propia en el estudio de los movimientos sociales, que integra elementos de la tradición del pensamiento crítico propio de la región con categorías provenientes de las tradiciones europea y norteamericana.

El presente artículo se focaliza en el análisis de las miradas críticas que se han desarrollado en las últimas dos décadas en América Latina para el estudio de los movimientos sociales. Hablamos aquí de perspectivas críticas para referir a aquellas que piensan los movimientos sociales en relación con el contexto en el que son producidos. Es decir, nos referimos a aquellos abordajes que incorporan a América Latina como perspectiva de análisis (Falero, 2020) y no como mera delimitación geográfica de lo que se pretende estudiar, reconociendo sus singularidades como constitutivas de los procesos colectivos que en ella se gestan. Esto implica analizar tanto aquellos aportes que retoman elementos de 
una tradición de pensamiento propia de la región como los trabajos que dialogan críticamente con esquemas teóricos surgidos en otras latitudes.

Si se toma este punto de partida, uno de los elementos comunes en estos enfoques es la crítica que sostienen al eurocentrismo implícito en la utilización mecánica de teorías y conceptos formulados para pensar otros contextos, porque esto supone un desconocimiento de los rasgos que históricamente han caracterizado a América Latina. En efecto, pensar la especificidad de la movilización social desplegada en las últimas décadas en nuestro continente implica analizarla en relación con los múltiples despojos de los que la región ha sido objeto en el contexto neoliberal (Seoane, Taddei y Algranati, 2011). Este rasgo es una de las claves para interpretar los procesos de movilización y cambio social, al delinear los ejes en torno a los cuales se configuran las luchas y los sujetos que se movilizan.

Por otra parte, siguiendo a Svampa (2009), puede decirse que otro elemento central en las teorías críticas de los movimientos sociales es el compromiso como aspecto indisociable de la actividad intelectual. El "cambio de época" que se procesa a partir de la década del 2000 ha habilitado, según la autora, una reconstrucción de la relación entre academia y compromiso político, la cual había sido anulada durante los años noventa con la profesionalización del saber académico y el repliegue del intelectualintérprete. Este cambio da lugar a la emergencia de lo que Svampa llama el "carácter anfibio" del investigador-intelectual, ${ }^{13}$ especialmente en el campo de los movimientos sociales.

Más allá de algunos rasgos comunes, estos abordajes son profundamente heterogéneos si se atiende a sus matrices teóricas. Uno de los aspectos que reflejan esta diversidad tiene que ver con los distintos niveles de diálogo que mantienen con las teorías clásicas sobre movimientos sociales. En un extremo, se identifican lecturas con una fuerte influencia del marxismo crítico, que colocan a las luchas sociales como centro del análisis y se distancian de las teorías que han hegemonizado los estudios sobre movimientos sociales (Modonesi, 2013), llegando a romper con el propio concepto de movimiento social (Gutiérrez, 2017). En el otro, se observan posiciones que proponen retomar los enfoques clásicos en esta materia, pero estableciendo un diálogo crítico con ellos, que ponga en el centro la especificidad latinoamericana (Bringel, 2011; Svampa, 2009). Este es uno de los elementos que dificulta el poder hablar de un paradigma latinoamericano, es decir, de un cuerpo teórico sistemático y coherente.

13 Esta noción supone la integración del modelo del investigador académico y el investigador militante sin desnaturalizar uno ni otro, conjugando ambas figuras en un solo paradigma, el del intelectual-investigador como anfibio. Del mismo modo que los anfibios pueden vivir en ambientes diferentes sin cambiar por ello su naturaleza, el investigador-intelectual anfibio puede habitar múltiples espacios combinando la crítica académica con el compromiso militante. 
Finalmente, debe señalarse que los estudios sobre movimientos sociales en este período han alumbrado nuevos debates y conceptos que brindan herramientas para el análisis de los procesos en cuestión. Uno de los más relevantes es el que refiere a la territorialidad como elemento constitutivo de los movimientos sociales. Tanto en lo urbano como en lo rural, el territorio aparece como espacio de resistencia y también como ámbito de creación de nuevas relaciones sociales. Esto ha llevado a muchos investigadores a entender los movimientos sociales latinoamericanos como movimientos socioterritoriales (Modonesi e Iglesias, 2016; Seoane, Taddei y Algranati, 2011; Svampa, 2009).

Otro de los debates relevantes del período es el que atañe a los nuevos sujetos que irrumpieron en la escena política a partir de la década del 2000. Svampa (2009) refiere a este punto aludiendo al carácter plebeyo de las formas de participación popular en el espacio público, siguiendo la propuesta de García Linera (2008). Lo plebeyo supone aquí una reivindicación de la cultura popular, negada y excluida, y una crítica a la cultura dominante. Por su parte, Modonesi e Iglesias (2016) colocan la noción de comunidad como concepto político que permite explicar el protagonismo de los movimientos indígenas en este ciclo, articulando la idea de comunidades campesinas e indígenas con la defensa de lo común. La noción de comunidad aparece, entonces, como horizonte político de las luchas registradas en la década del 2000 (Gutiérrez, 2008), vinculando su carácter antineoliberal con el anhelo de otro modo de existencia centrado en la valorización de la vida y de la naturaleza.

Por otra parte, el concepto de autonomía ha sido una de las claves teóricas en el análisis de los movimientos sociales que caracterizan el nuevo milenio. De acuerdo con Modonesi e Iglesias (2016), la idea de autonomía se plantea en los estudios contemporáneos haciendo referencia a dos dimensiones: por un lado, como independencia de los partidos políticos y los gobiernos, y por tanto como la preservación de espacios de decisión propios de los movimientos; por otro lado, la autonomía aparece como la capacidad de los movimientos de producir sus propias condiciones de vida, resolviendo en forma autogestionaria necesidades como la educación, la salud y la vivienda. En este sentido, los movimientos latinoamericanos son teorizados como espacios capaces de producir nuevas relaciones sociales, impulsando transformaciones que van más allá de lo estatal.

\section{La formación, la gran ausente}

Resulta llamativo el hecho de que, en las distintas revisiones teóricas que documentan el desarrollo de los estudios sobre movimientos sociales en las últimas décadas, la formación como eje del debate resulte la gran ausente. $\mathrm{Ni}$ los trabajos de Modonesi e Iglesias (2016), ni los de Seoane, Taddei y Algra- 
nati (2011), ni los de Svampa (2009) colocan a la formación como una de las discusiones relevantes del período. ${ }^{14}$ Esto contrasta con la importancia que el tema ha tenido, tanto en la construcción de los movimientos sociales en la región como en la producción teórica y empírica.

En América Latina, los estudios centrados en la relación entre formación y movimientos sociales comenzaron a desarrollase a principios de este siglo, buscando dar cuenta de las prácticas educativas desplegadas por diversos movimientos. Una de las experiencias pioneras en este sentido es la del Movimiento de los Trabajadores Rurales Sin Tierra (MST) en Brasil, que desde hace décadas sostiene espacios educativos propios. Enfocándose en esta experiencia, uno de los primeros estudios que analiza la relación entre movimientos sociales y educación es el de Caldart (2004). Para esta autora, el MST puede concebirse en su totalidad como un principio educativo, en el que las diversas actividades contienen una dimensión pedagógica (la lucha, la organización, el trabajo con la tierra, los espacios educativos). Otro de los trabajos destacados en este período es el de Michi (2010), que analiza la relación entre movimientos campesinos y educación a partir del estudio del MST y del Movimiento Campesino de Santiago del Estero (MOCASE), en Argentina. Para la autora, la acción pedagógica de los movimientos sociales, entendida en un sentido amplio como producción de cultura, forma parte del proceso de organización, de apropiación territorial y de politización.

En los años posteriores, comenzaron a aparecer diversos estudios que analizaban las prácticas formativas de movimientos sociales de base urbana, teniendo como uno de sus focos la experiencia piquetera en Argentina y las organizaciones territoriales que se consolidaron en los años posteriores, así como también espacios formativos vinculados a empresas recuperadas por sus trabajadores (Aguiló y Wahren, 2014; Elisalde, 2008; Palumbo, 2017). En Uruguay, se han realizado estudios regionales comparados que incluyen distintos tipos de movimientos de base urbana y rural (Falero et al., 2011) y trabajos centrados en casos específicos, que abarcan desde cooperativas de vivienda hasta organizaciones socioproductivas (Menéndez, 2014; Sarachu, 2013).

Si se revisan estos trabajos, resulta evidente que se ha conformado un importante campo de estudios sobre el tema, que articula acumulación teórica y empírica, el cual se ha consolidado acompañando el desarrollo de las experiencias educativas de los movimientos sociales. Siguiendo a Palumbo (2016), puede decirse que la educación es una de las dimensiones

14 Cabe aclarar que existen investigaciones que destacan el componente formativo como una característica específica de los movimientos sociales latinoamericanos que emergieron desde fines de la década del noventa (véase, por ejemplo, Zibechi, 2003). El planteo aquí incluido refiere, en cambio, al hecho de que los trabajos que sistematizan la producción teórica de las últimas décadas sobre movimientos sociales en América Latina no colocan la relación entre movimientos sociales y educación como una de las aperturas teóricas relevantes del período. 
constitutivas de los movimientos populares latinoamericanos. En estos ámbitos se ensayan nuevos formatos y pedagogías, circulan otros saberes y se conforman prácticas prefigurativas que afirman en el presente la posibilidad de nuevos proyectos a futuro.

En forma paralela a la importancia que la formación ha tenido en la construcción de los movimientos sociales latinoamericanos en las últimas décadas, este breve repaso permite afirmar la relevancia teórica que este debate ha tenido en la elaboración de una mirada latinoamericana sobre el tema, abonando algunos de los debates teóricos más relevantes del período.

Las prácticas formativas de los movimientos sociales están ligadas a su carácter territorial, al aparecer el territorio como central a la hora de articular demandas, luchas y prácticas con la formación, tanto a nivel urbano como rural (Falero et al., 2011). Asimismo, estas constituyen un componente clave en las disputas territoriales que los movimientos sociales despliegan, en la medida en que suponen la producción de nuevos vínculos, la politización y la construcción de nuevos sentidos ligados a un territorio específico.

Adicionalmente, la formación puede entenderse como una dimensión central de las prácticas de construcción de autonomía desplegadas por los movimientos sociales, es decir, de sus capacidades de formular respuestas ante las problemáticas que atraviesan y, en el mismo proceso, elaborar horizontes alternativos de transformación social. Junto con las experiencias que dan respuestas materiales ante los despojos que los movimientos visibilizan (como la producción colectiva de alimentos, la construcción de viviendas o la creación de emprendimientos de trabajo autogestionado), la dimensión formativa supone la producción y la transmisión de un conjunto de significados que dan sentido a estas prácticas.

Conceptualizada de este modo, la dimensión formativa de los movimientos sociales permite debatir y complejizar los abordajes clásicos en este campo de estudios. Esta supone un giro teórico, que descentra la mirada de las actividades públicas y visibles, de la protesta como expresión de la actividad de los movimientos sociales — que fuera el centro de las teorías norteamericanas-, para colocarla en las prácticas de construcción subterránea que estos desarrollan. En tal sentido, permite complejizar la idea de latencia propuesta por Melucci (1999), entendida como momento de producción de redes y sentidos, contribuyendo a comprender sus múltiples dimensiones y niveles y a analizar el modo en que en ella se combinan componentes materiales y simbólicos.

\section{Conclusiones}

¿Asistimos actualmente a una renovación teórica en el campo de los estudios sobre movimientos sociales en América Latina? ¿Cuáles son sus características? ¿Qué lugar ocupa el debate sobre la formación en este proceso? 
Como se ha fundamentado a lo largo de este artículo, en las últimas dos décadas se ha desarrollado en América Latina una importante producción académica orientada al análisis de los movimientos sociales que han emergido en la región, la cual articula elaboración teórica y acumulación empírica. Consideramos que esta supone una profunda renovación teórica en el campo de los estudios sobre movimientos sociales y acciones colectivas, en la medida en que implica una crítica a las teorías dominantes en esta materia, un análisis de los movimientos sociales emergentes en relación con las características de su contexto, desarrollos conceptuales originales y nuevas articulaciones de abordajes clásicos.

Distintos investigadores han sistematizado algunos de los debates más relevantes del período, como son los que refieren a la autonomía, la territorialización, la irrupción política de nuevos sujetos y la emergencia de nuevos horizontes políticos. Sostenemos aquí que el debate acerca de la relación entre movimientos sociales y formación constituye una de las claves teóricas para captar la singularidad de las luchas sociales que se han registrado en este período en la región.

La discusión acerca de los alcances de esta renovación aún está abierta. En tal sentido, Modonesi e Iglesias (2016) señalan que, si bien se reconocen planteamientos teóricos interesantes que impugnan las teorías clásicas sobre la acción colectiva y abren campos de reflexión fértiles, estos no se han conformado como un paradigma alternativo capaz de desplazar a los dominantes. La perspectiva aquí propuesta coincide con esta apreciación general, ya que la heterogeneidad de las elaboraciones teóricas registradas y su falta de sistematicidad impiden colocarlas como un paradigma articulado y coherente.

Sin embargo, se sostiene aquí que la ausencia de un desarrollo teórico de tales características no anula la importancia de la producción reciente en torno al tema que nos ocupa. Si bien no se han constituido como un nuevo paradigma capaz de desplazar a los anteriores, estas teorizaciones han interpelado su capacidad interpretativa, produciendo nuevas elaboraciones y reformulaciones conceptuales. Siguiendo esta línea, entendemos que uno de los giros teóricos más interesantes del período es la construcción de un pensamiento situado en la propia realidad latinoamericana, es decir, que da centralidad a las características del contexto, reconociendo, a su vez, sus vínculos con procesos y relaciones más generales (Falero, 2020).

Finalmente, cabe señalar algunos de los límites de estos desarrollos. Sostenemos que estas teorizaciones se encuentran muy ligadas a experiencias de lucha emergidas en los primeros años del siglo XXI, signadas por el protagonismo de las comunidades indígenas en países como Bolivia y Ecuador y por grandes movilizaciones urbanas, como fue la de 2001 en Argentina, así como la experiencia piquetera que floreció en los años posteriores. 
Hoy, casi dos décadas después, el contexto es otro. En la actualidad, las luchas más dinámicas parecen surgir de articulaciones entre movimientos feministas, luchas indígenas y movilizaciones de jóvenes urbanos, que rebasan y desbordan las organizaciones sociales "clásicas". Esto se da en un contexto político caracterizado por el declive del ciclo progresista y el ascenso de sectores de derecha ultraconservadora. Será la tarea de los próximos años pensar nuevas ideas que permitan captar estas transformaciones.

\section{Referencias bibliográficas}

Aguiló, V. y J. Wahren (2014). Los bachilleratos populares de Argentina como “campos de experimentación social”. Argumentos, 27(74), pp. 97-114.

Almeida, P. y A. Cordero (eds.) (2017). Movimientos sociales en América Latina: perspectivas, tendencias y casos. Buenos Aires: CLACSO.

Alonso, A. (2009). As teorias dos movimentos sociais: um balanco do debate. Lua Nova, 76, pp. 49-86.

Benford, R. y D. Snow (2000). Framing processes and social movements: An overview and assessment. Annual Review of Sociology, 26, pp. 611-639.

Bringel, B. (2011). A busca de uma nova agenda de pesquisa sobre os movimentos sociais e o confronto político: diálogos con Sidney Tarrow. Politica y sociedade, 10(18), pp. 51-73.

Caldart, R. (2004). Pedagogia do Movimento Sem Terra. San Pablo: Expressao Popular.

Calderón, F. (1986). Los movimientos sociales ante la crisis. Buenos Aires: UNU-CLACSO- IISUNAM.

Camacho, D. (1987). Movimientos sociales, algunas definiciones conceptuales. Revista de Ciencias Sociales de la Universidad de Costa Rica, 37-38, pp. 7-21.

Cohen, J. (1985). Strategy or identity: new theoretical paradigms and contemporary social movements. Social Research, 52(4), pp. 663-716.

Diani, M. (2015). Revisando el concepto de movimiento social. Encrucijadas, 9, pp. 1-16.

Della Porta, D. y Diani, M. (2015). Los movimientos sociales. Madrid: Universidad Complutense-Centro de Investigaciones Sociológicas. 
Elisalde, R. (2008). Movimientos sociales y educación: bachilleratos populares en empresas recuperadas y organizaciones sociales. Experiencias pedagógicas en el campo de la educación de jóvenes y adultos. En R. Elisalde y M. Ampudia (comps.) (2008). Movimientos sociales y educación: teoría e historia de la educación popular en Argentina y América Latina. Buenos Aires: Buenos Libros, pp. 67-99.

Falero, A. (2018). El tratamiento conceptual del sujeto colectivo en América Latina: de lo obvio a lo problemático. El Uruguay desde la sociología XVI. Montevideo: Departamento de Sociología, Facultad de Ciencias Sociales, Universidad de la República, pp. 25-39.

Falero, A.; A. Casas; A. Rieiro y B. Rocco (2011). Movimientos sociales y formación político técnica: reflexiones a partir de cinco casos en América Latina. En Y. Acosta; A. Falero; A. Rodríguez; I. Sans y G. Sarachu (coords.) (2011). Pensamiento crítico y sujetos colectivos en América Latina: perspectivas interdisciplinarias. Montevideo: Trilce, pp. 351-371.

Falero, A. (2020). América Latina: entre perspectiva de análisis y proyecto sociopolítico. En E. Torres (ed.) (2020). Hacia la renovación de la teoría social latinoamericana. Buenos Aires: CLACSO, pp. 153-182.

García Linera, A. (2008). La potencia plebeya. Acción colectiva a identidades indigenas, obreras y populares en Bolivia. Buenos Aires: CLACSOPrometeo.

Gohn, M. G. (1997). Teorías dos movimentos sociais. Paradigmas clássicos e contemporâneos. San Pablo: Loyola.

Gohn, M. G. (2008). Abordagens teóricas no estudo dos movimentos sociais na América Latina. Caderno CRH, 21(54), pp. 439-455.

Gutiérrez, R. (2008). Los ritmos del Pachakuti. Movilización y levantamiento popular-indígena en Bolivia (2000-2005). Buenos Aires: Tinta Limón.

Gutiérrez, R. (2017). Horizontes comunitario-populares. Producción de lo común más allá de las políticas estado-céntricas. Madrid: Traficantes de Sueños.

McAdam, D.; J. McCarthy y M. Zald (1999). Oportunidades, estructuras de movilización y procesos enmarcadores: hacia una perspectiva sintética y comparada de los movimientos sociales. En D. McAdam; J. McCarthy y M. Zald (eds.) (1999). Movimientos sociales: perspectivas comparadas. Madrid: Istmo, pp. 21-46. 
McAdam, D.; S. Tarrow y C. Tilly (2001). Dynamics of contention. Nueva York: Cambridge University Press.

McAdam, D.; S. Tarrow y C. Tilly (2009). Para mapear o confronto político. Lua Nova, 76, pp. 11-48.

McAdam, D. y S. Tarrow (2011). Introduction: Dynamics of contention ten years on. Mobilization, 16(1), pp. 1-10.

Melucci, A. (1994). Asumir un compromiso: identidad y movilización en los movimientos sociales. Zona Abierta, 69, pp. 153-180.

Melucci, A. (1999). Acción colectiva, vida cotidiana y democracia. México DF: El Colegio de México, Centro de Estudios Sociológicos.

Menéndez, M. (2014). Educación en movimiento: la experiencia de la Federación Uruguaya de Cooperativas de Vivienda por Ayuda Mutua [Tesis de Maestría en Psicología Social]. Montevideo: Facultad de Psicología, Universidad de la República.

Michi, N. (2010). Movimientos campesinos y educación. Estudio sobre el Movimiento de Trabajadores Rurales Sin Tierra y el Movimiento Campesino de Santiago del Estero MOCASE-VC. Buenos Aires: El Colectivo.

Modonesi, M. (2013). Marxismo crítico y teorías de los movimientos sociales [en línea]. Disponible en: <https://www.yumpu.com/es/document/ $\mathrm{read} / 36711725 /$ marxismo-critico-y-teorias-movimientos $>$ [acceso: $18 \mathrm{de}$ junio de 2019].

Modonesi, M. y M. Iglesias (2016). Perspectivas teóricas para el estudio de los movimientos sociopolíticos en América Latina: ¿cambio de época o década perdida? De Raíz Diversa, 3(5), pp. 95-124.

Palumbo, M. (2016). Educación en movimientos populares rurales: un estado del arte. Revista historia de la educación latinoamericana, 18(26), pp. 219-240.

Palumbo, M. (2017). Dinámicas de construcción de subjetividades políticas y epistémicas en dispositivos pedagógicos de formación política. Un estudio en movimientos populares multisectoriales del Área Metropolitana de Buenos Aires (2011-2015) [Tesis de Doctorado en Educación]. Buenos Aires: Facultad de Filosofía y Letras, Universidad de Buenos Aires.

Sarachu, G. (2013). Trabajo, educación y formación: itinerarios compartidos con las organizaciones autogestionarias socio-productivas desde la extensión universitaria. En R. Elisalde; N. M. Dal Ri; M. Ampudia; A. Falero y K. 
Pereyra (comps.) (2013). Movimientos sociales, educación popular y trabajo autogestionado en el cono sur. Buenos Aires: Buenos Libros, pp. 107-134.

Schuster, F.; G. Pérez; S. Peryra; M. Armesto; M. Armelino; A. García; A. Natalucci; M. Vázques y P. Zipcioglu (2006). Transformaciones de la protesta social en Argentina 1989-2003. Documento de Trabajo. Buenos Aires: IIGG-UBA.

Seoane, J.; E. Taddei y C. Algranati (2011). El concepto de "movimiento social" a la luz de los debates y la experiencia latinoamericana recientes. Controversias y Concurrencias Latinoamericanas, 4 [en línea]. Disponible en: <https:// www.cetri.be/IMG/pdf/090113_EL_CONCEPTO_DE_MOVIMIENTO_ SOCIAL_A_LA_LUZ_DE_LOS_DEBTES_Y_LA_EXPERIENCIA_ LATINOAMERICANA_RECIENTE_VERSION_FINAL_G.pdf> [acceso: 18 de junio de 2020].

Svampa, M. (2008). Cambio de época. Movimientos sociales y poder político. Buenos Aires: Siglo XXI-CLACSO.

Svampa, M. (2009). Protesta, movimientos sociales y dimensiones de la acción colectiva en América Latina. Trabajo presentado en Jornadas de Homenaje a C. Tilly, Universidad Complutense de Madrid-Fundación Carolina, 7-9 de mayo de 2009 [en línea]. Disponible en: <http://www.maristellasvampa.net/ archivos/ensayo57.pdf $>$ [acceso: 18 de junio de 2020].

Tarrow, S. (2009). El poder en movimiento. Los movimientos sociales, la acción colectiva y la política. Madrid: Alianza.

Touraine, A. (1990). Movimientos sociales de hoy. Actores y analistas. Debates con Alberto Melucci y otros. Barcelona: Editorial Hacer.

Zibechi, R. (2003). Los movimientos sociales latinoamericanos: tendencias y desafíos. $O S A L, 9$, pp. 185-188.

\section{Contribución de autoría}

El trabajo en su totalidad fue realizado por Mariana Fry. 